\title{
Endoscopic corpus callosotomy and hemispherotomy
}

\author{
Sandeep Sood, MD, Neena I. Marupudi, MD, MS, Eishi Asano, MD, Abilash Haridas, MD, and \\ Steven D. Ham, DO
}

Department of Pediatric Neurosurgery, Children's Hospital of Michigan, Detroit, Michigan

\begin{abstract}
OBJECT Corpus callosotomy and hemispherotomy are conventionally performed via a large craniotomy with the aid of a microscope for children with intractable epilepsy. Primary technical considerations include completeness of disconnection and blood loss. The authors describe an endoscopic technique performed through a microcraniotomy for these procedures.
\end{abstract}

\begin{abstract}
METHODS Four patients with drop attacks and 2 with intractable seizures related to a neonatal stroke underwent endoscopic complete corpus callosotomy and hemispherotomy, respectively. The surgeries were performed through a 2- to 3-cm precoronal microcraniotomy. Interhemispheric dissection to the corpus callosum was done using the standard technique. Subsequently, the bimanual technique with a suction device mounted on an endoscope was used to perform a complete corpus callosotomy, including interforniceal and anterior commissure disconnection. In patients who had hemispherotomy, the fornix was resected posteriorly and lateral disconnection was done by unroofing the temporal horn. Anteriorly, endoscopic corticectomy was done along the ipsilateral anterior cerebral artery to reach the bifurcation of the internal carotid artery to complete the anterior disconnection. Postoperative MRI and diffusion tensor imaging (DTI) of the brain were performed to confirm complete disconnection.
\end{abstract}

RESULTS The procedure was accomplished successfully in all patients, with excellent visualization secured. None of the patients required a blood transfusion. Postoperative MRI and DTI confirmed completeness of the disconnection. Patients who underwent corpus callosotomy had complete resolution of drop attacks at a mean follow-up of 6 months, and patients who underwent hemispherotomy became seizure free.

CONCLUSIONS Endoscopic corpus callosotomy and hemispherotomy are surgically feasible procedures associated with minimal blood loss, minimal risk, and excellent visualization.

http://thejns.org/doi/abs/10.3171/2015.5.PEDS1531

KEY WORDS endoscopic surgery; hemispherotomy; corpus callosotomy; seizures; hemispherectomy; epilepsy; technique

$\mathrm{D}$ ISCONNECTIVE procedures such as corpus callosotomy and hemispherotomy are cornerstones of surgical management of intractable epilepsy in children. These procedures have stood the test of time and have been traditionally done through a large craniotomy with the aid of a microscope. The major disadvantages of an open procedure include increased blood loss, postoperative pain, and a longer hospital stay. Bahuleyan et al. and others have described the feasibility of using an endoscope for corpus callosotomy in cadavers. ${ }^{1,2,6,13}$ We have recently refined our bimanual endoscopic technique, as previously described for resection of intraventricular and pineal tumors, ${ }^{11,12}$ and extended it to corpus callosotomy and hemispherotomy.

\section{Methods}

Over a 1-year period, 4 patients underwent a complete corpus callosotomy and 2 underwent a hemispherotomy performed using the bimanual endoscopic technique (Table 1). The mean age of the patients was 11 years (SD 6.9 years; range $2.5-20$ years). All patients had medically in-

ABBREVIATIONS ACA = anterior cerebral artery; CUSA = Cavitron ultrasonic aspirator; DTI = diffusion tensor imaging; EEG = electroencephalography; $\mathrm{MCA}=$ middle cerebral artery. 
TABLE 1. Demographic data in 6 patients who underwent endoscopic disconnection for intractable epilepsy

\begin{tabular}{cclccc}
\hline Case No. & Age (yrs), Sex & \multicolumn{1}{c}{ Procedure } & Duration (hrs) & Blood Loss (ml) & Transfusion \\
\hline 1 & $12, \mathrm{M}$ & Corpus callosotomy $^{*}$ & 3 & $<50$ & None \\
\hline 2 & $18, \mathrm{~F}$ & Posterior corpus callosotomy $^{*}$ & 3.5 & 80 & None \\
\hline 3 & $5, \mathrm{M}$ & Corpus callosotomy $^{*}$ & 2.5 & $<50$ & None \\
\hline 4 & $9, \mathrm{M}$ & Corpus callosotomy & 2 & $<50$ & None \\
\hline 5 & $20, \mathrm{~F}$ & Lt hemispherotomy & 5 & $<50$ & None \\
\hline 6 & $2.5, \mathrm{M}$ & Rt hemispherotomy & 4 & $<50$ & None \\
\hline
\end{tabular}

* Patients had a complete corpus callosotomy, forniceal disconnection, and disconnection of the anterior commissure.

$\dagger$ Patient previously had an anterior two-thirds corpus callosotomy.

tractable seizures and underwent extensive workup with video electroencephalography (EEG), MRI, and PET prior to being considered for a disconnective surgical procedure. Both patients who underwent hemispherotomy had suffered neonatal strokes involving the middle cerebral artery (MCA), with an area of encephalomalacia. A postoperative MRI study with diffusion tensor imaging (DTI) was obtained in each patient to confirm the extent of disconnection.

The equipment used comprised an endoscope cannula mounted on a standard $10-\mathrm{F}$ suction device by using a coupling clamp, and was secured as shown in Fig. 1. The suction is below the endoscope cannula and extends $2 \mathrm{~cm}$ beyond it to take advantage of the natural surgical corridor. A $0^{\circ}$, high-definition, rigid rod lens Wolfe endoscope was then inserted through the cannula for use. This was held in the left hand just as a regular suction piece would be used during routine surgery. The right hand was free to use a second instrument for dissection or coagulation.

Each patient was positioned supine and the head was secured in pin fixation (Fig. 1). Under MRI-guided neuronavigation, a 2- to $3-\mathrm{cm}$ incision was marked just lateral to the midline anterior to the coronal suture to avoid the

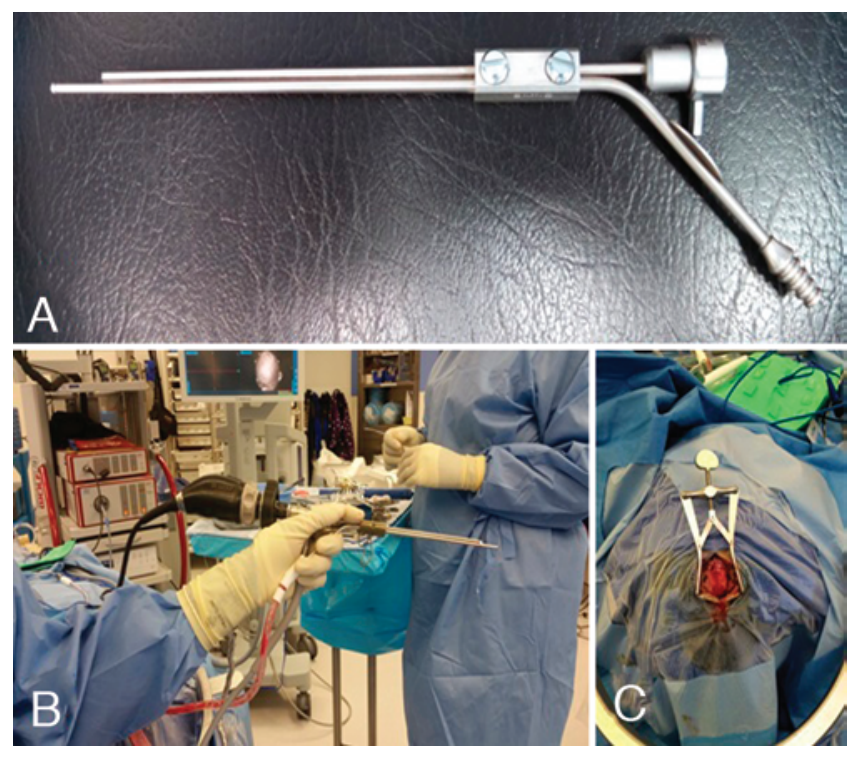

FIG. 1. A: Suction cannula mounted on an endoscope cannula using a clamp. B: Endoscope with mounted suction is held in the operator's left hand. C: Operative position and 2- to $3-\mathrm{cm}$-long precoronal incision. Figure is available in color online only. bridging veins and provide the best trajectory for callosotomy, as determined on sagittal MRI. A precoronal 2- to 3 -cm-diameter craniotomy was made just lateral to the superior sagittal sinus in front of the bridging vein. The dura mater was opened with the flap based medially. Initial dissection to the corpus callosum was done under loupe magnification to expose $2 \mathrm{~cm}$ of the corpus callosum surface in the anterior third of the corpus callosum. Self-retaining retraction with the Budde Halo retractor system was used on the ipsilateral hemisphere. The subsequent procedure was assisted by retractor and carried out under visualization from the endoscope equipped with mounted suction for the bimanual technique, using dissectors, Cavitron ultrasonic aspirator (CUSA), or standard bipolar cautery as needed.

Figure 2 shows the steps involved in complete corpus callosotomy with forniceal disconnection and disconnection of the anterior commissure. The surface of the corpus callosum is incised with the bipolar. Using the CUSA, the corpus callosum is removed in the midline to identify the folds of the septum pellucidum. The resection of the corpus callosum is continued anteriorly between the 2 anterior cerebral arteries (ACAs) to reach the genu of the corpus callosum. From here, the resection is continued inferiorly to visualize the ACA on the undersurface of the corpus callosum and then traced posteriorly to the level of the rostrum. At this point the posterior resection of the corpus callosum is started, using the suction on the endoscope to retract the pericallosal arteries superiorly. In that way, the CUSA can be used to remove the corpus callosum to reach the curve of the splenium, and thence on to the septum interpositum containing the internal cerebral veins. Proceeding anteriorly on the septum pellucidum, removing the overlying corpus callosum, as the commissure of the fornix is split in the midline, the appearance of choroid plexus marks the opening of the foramen of Monro. Anteriorly, the anterior commissure is visualized and can be cut with the bipolar or CUSA. Any bleeding can be easily controlled with the bipolar.

For hemispherotomy (Fig. 3, Video 1), first the anterior corpus callosotomy is done to expose the ACA to the rostrum.

VIDEO 1. Clip showing the steps in endoscopic hemispherotomy performed using the bimanual technique with the aid of an endoscope mounted on a suction device. Copyright Sandeep Sood. Published with permission. Click here to view with Media Player. Click here to view with Quicktime.

Next, using the CUSA, a corticectomy over the ACA is 


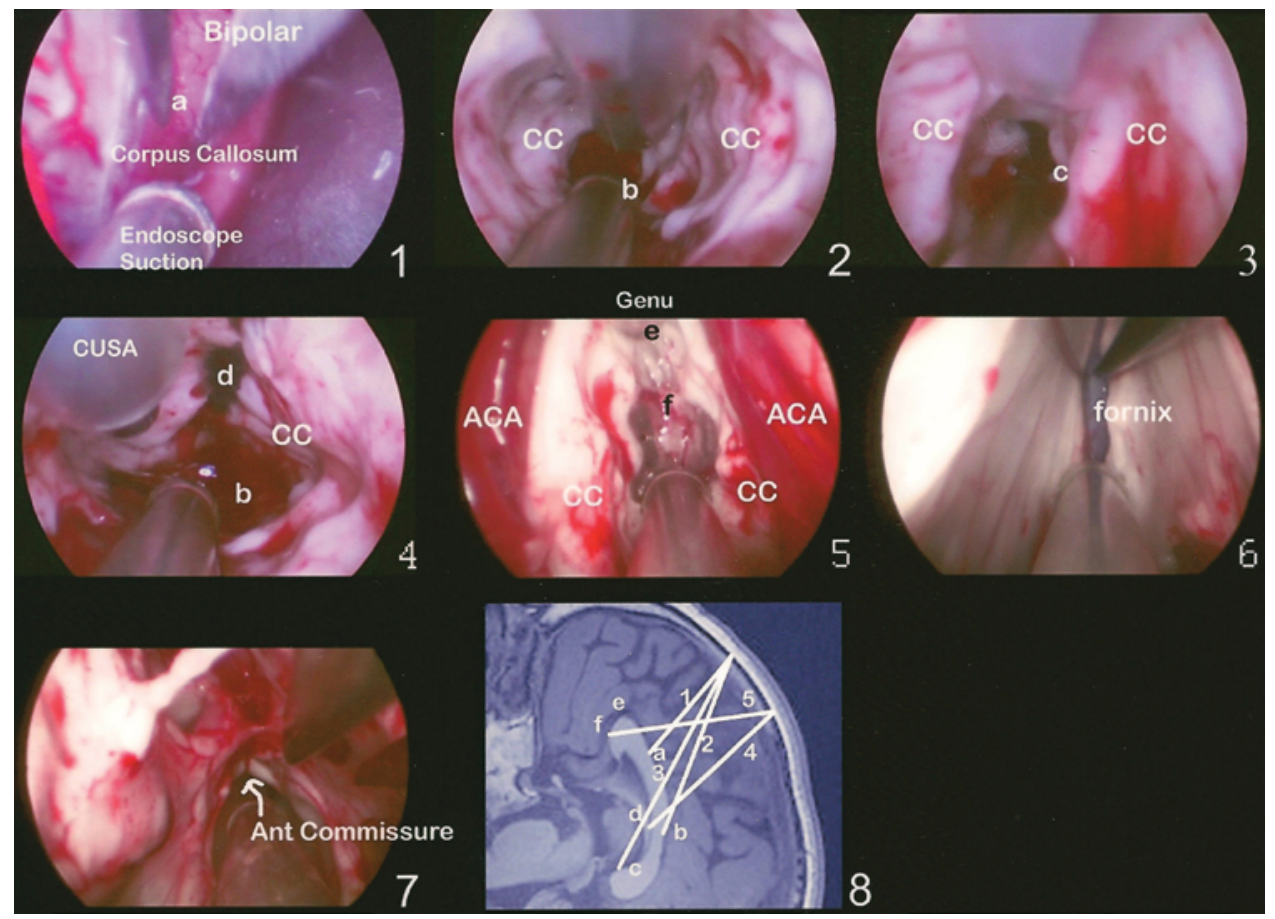

FIG. 2. Operative images of endoscopic corpus callosotomy. Panels 1-5 represent the views along the corresponding trajectories in panel 8, the sagittal CT scan; letters a-f in panels $1-5$ correspond to the same letters in panel 8; panels 6 and 7 show fornix disconnection and anterior (ant) commissure disconnection. CC = corpus callosum. Figure is available in color online only.

carried laterally on through the floor of the frontal horn onto the first part of MCA, completing the anterior or frontobasal disconnection. Care must be taken to get to the midline without missing the most anterior fibers of the rostrum. At this point attention is directed to the atrium, where the choroid plexus is followed anteriorly to make a corticectomy lateral to the choroid plexus with the CUSA under endoscopic vision and to unroof the temporal horn from back to front, along the thalamostriatal groove of the lateral ventricle, until the MCA is seen at the anterior inferior extent of the corticectomy. This results in separation of the basal ganglia and the overlying insula from the thalamus, completing the lateral disconnection. Finally, the corpus callosotomy is completed posteriorly and the fornix is cut over the thalamus in the ventricular trigone.

Even though the hippocampus and amygdala are left intact, their hemispheric connection through the temporal lobe is effectively interrupted by the callosotomy. The only other efferent from the hippocampus is the fornix, and it is cut, as stated above, in the region of the ventricular trigone. The amygdala complex has 2 other major efferents: the stria terminalis along the tail of the caudate, and the diagonal band of Broca. The stria terminalis and fibers of anterior commissure lie in the roof of the temporal horn, just lateral to the choroid fissure. The unroofing of the temporal horn just lateral to the choroidal fissure over the choroid plexus effectively disconnects this fiber system. Finally, the diagonal band of Broca from the amygdala runs over the MCA, just anterior to the optic tracts, on the anterior perforated substance. Corticectomy to expose the entire ACA over to the MCA sections this band, de-efferentating the amygdala.

\section{Results}

The operation was accomplished successfully in all patients. In 2 patients, the operating microscope was briefly used to confirm the anatomy. The operating time for endoscopic corpus callosotomy was $2-3$ hours. The operating time for endoscopic hemispherotomy was $4-5$ hours. There was minimal blood loss and none of the patients required a blood transfusion. The median hospital stay was 4 days.

Patients who underwent hemispherotomy have continued to be seizure free, and those who underwent corpus callosotomy have had no drop attacks at a mean followup of $6 \pm 2.1$ months (Table 2). Postoperative DT images (Fig. 4) showed complete disconnection in those patients in whom these procedures were done.

\section{Discussion}

Traditionally, endoscopic procedures have been done using a channeled endoscope placed through a bur hole. In this so-called 1-handed technique, an instrument is used through the channel of the endoscope for biopsy or piecemeal removal of the lesion (Fig. 5). The main disadvantage is that the ability to dissect is limited by the movement of the instrument only in the axial direction. Furthermore, bleeding from the lesion may be difficult to control because of limitation of the endoscopic bipolar or unipolar.

An alternative method that can be performed using an endoscope is through a microcraniotomy? The endoscope is held in position either manually by an assistant or with a holder. The surgeon uses regular instruments and is able to bimanually dissect the lesion using standard microsurgi- 


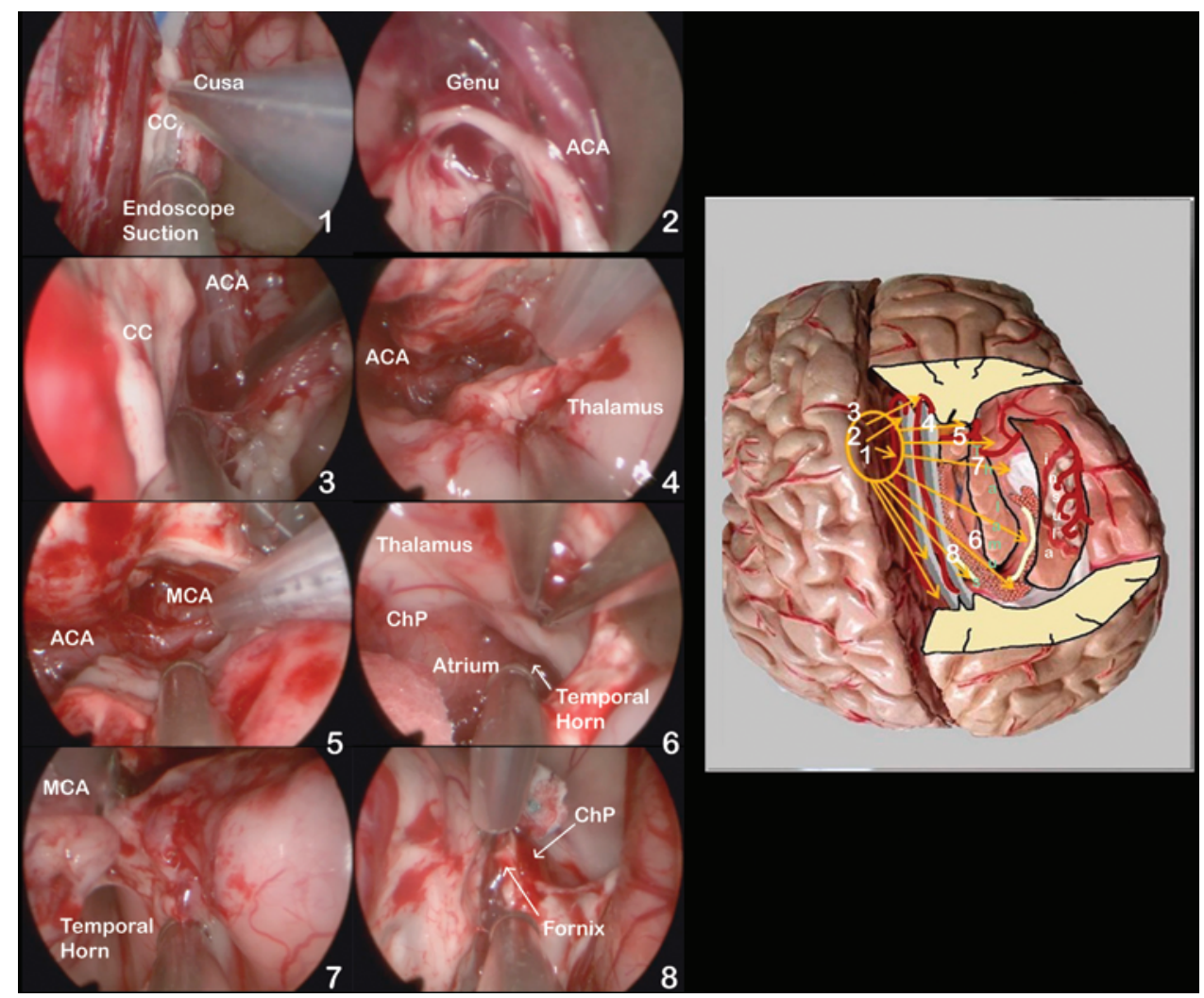

FIG. 3. Intraoperative images of endoscopic hemispherotomy. Panels 1-8 represent the views along the corresponding trajectories, with the cut section of the brain on the right: panels $1-3$, anterior corpus callosotomy; 4 and 5 , anterior frontobasal disconnection; 6 and 7, lateral disconnection; 8, fornix disconnection; and rightmost panel, posterior corpus callosotomy. ChP = choroid plexus.

cal techniques. We refer to this as a 3-handed technique, and it has become a very standard approach for transsphenoidal or transnasal basal approaches. ${ }^{10}$ The advantages are a smaller incision and less retraction. The disadvantage is that every time the field of surgery has to change, the endoscope has to be moved to a new position. This can be somewhat tedious and frustrating in long operations. In addition, crowding of instruments through a small opening often results in an irritating instrument "scissoring."

We have previously described removal of deep thalamic, intraventricular, and pineal tumors with a bimanual technique (which we also refer to as the 2-handed technique) by using an endoscope with mounted suction in the left hand, and an instrument in the right hand allowing for bimanual dissection, use of regular bipolar cautery, and dissecting instruments. ${ }^{11,12}$ Cutler et al. ${ }^{4}$ have used a custom-made suction device with an endoscope for vestibular neurectomies performed using the same technique. This retains the advantages of microsurgical techniques and allows for the use of microcraniotomy, with advantages of a smaller incision and minimal retraction, and it avoids instrument scissoring.

Bahuleyan et al. ${ }^{2}$ and Guerrero and Cohen ${ }^{6}$ have described the feasibility of using endoscopes for corpus callosotomy, based on their use of a single-channel endoscope with a parasagittal bur hole in a cadaveric setting. However, the inability to effectively control bleeding in the event of a vascular injury is a major deterrent to use of this approach in a clinical setting. We see a similar disadvantage in using an eyebrow incision and the supraorbital trephination and endoscopic corpus callosotomy approach described by Tubbs et al. ${ }^{13}$

A standard complete corpus callosotomy is performed through a craniotomy that is approximately $8 \times 4 \mathrm{~cm}$, and

TABLE 2. Outcome of seizures following a complete corpus callosotomy and disconnection of interforniceal and anterior commissure*

\begin{tabular}{cclll}
\hline Case No. & Atonic Seizures (drop attack) & Generalized Seizures & Focal Seizures & Myoclonic Seizures \\
\hline 1 & Freedom & Freedom & Freedom & NA \\
\hline 2 & Freedom & Significantly reduced & Significantly reduced & NA \\
\hline 3 & Freedom & Freedom & NA & Significantly reduced $\dagger$ \\
\hline 4 & Freedom & Freedom & NA & Significantly reduced $\dagger$ \\
\hline
\end{tabular}

$\mathrm{NA}=$ not applicable.

* Outcome determined at a mean follow-up of $6 \pm 2.1$ months.

$\dagger$ Indicates $>90 \%$ reduction in seizure frequency. 

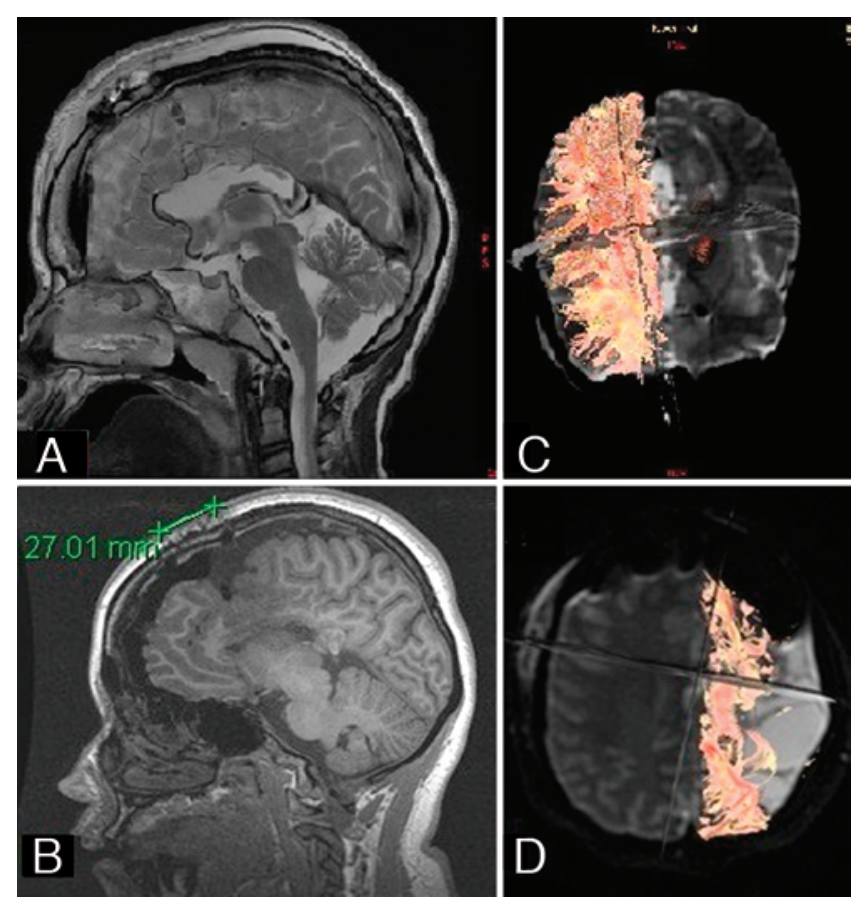

FIG. 4. Postoperative MRI scans obtained after (A) complete corpus callosotomy and (B) hemispherotomy, showing the precoronal microcraniotomy. Postoperative DTI studies demonstrating completeness of disconnection after (C) corpus callosotomy and (D) hemispherotomy. Figure is available in color online only.

generally half of it extends behind the coronal suture to provide access to the splenium. ${ }^{9}$ However, retraction of the posterior frontal lobe puts the bridging veins at risk for injury. Our endoscopic approach allows for a much smaller $(3 \times 2 \mathrm{~cm})$ precoronal craniotomy that is done anterior to the major bridging veins. Minimized retraction, improved visualization, and greater magnification with an endoscope make access to the splenium possible even from this far anterior approach, as has been previously demonstrated in cadaveric studies. ${ }^{2}$ Similarly, the improved visualization and magnification with the use of an endoscope is advantageous in confirming the completeness of lateral disconnection while performing the hemispherotomy.

Although as expected, the patients who underwent a hemispherotomy were seizure free, the complete cessation of drop attacks in patients who underwent complete corpus callosotomy is a better outcome than reported in literature. ${ }^{9}$ This may relate to a shorter follow-up period, but may be partly due to the fact that in addition to a complete corpus callosotomy, our patients also underwent disconnection of the interforniceal commissure and the anterior commissure. It has been our observation that intraoperatively monitored bilateral scalp EEG may not desynchronize until the interforniceal commissure and anterior commissure have been disconnected. A good seizure outcome in our patient cohort might also be attributed to the absence of cortical lesions on MRI, as previously reported. ${ }^{8}$

In this paper we have demonstrated that the endoscope with mounted suction can be used bimanually and effectively to perform disconnective procedures. The steps of surgery for corpus callosotomy are similar to those used in microsurgical approaches. Likewise, the steps of surgery for hemispherotomy are similar to those described by Delalande et al. ${ }^{5}$ for parasagittal vertical hemispherotomy, but without the need for posterior cortical resectionwhich is done to improve exposure to the ventricular trigone during surgery using a microscope. Additionally, the improved visualization with the endoscope allows the use of a precoronal microcraniotomy without the need for disruption of the parasagittal draining veins. These vessels are encountered in a posterior frontal craniotomy that is used for a microsurgical corpus callosotomy or parasagittal vertical hemispherotomy of Delalande. Needless to say, some cadaver experience or familiarity with the use of endoscopes and significant experience with microsurgical vertical hemispherotomy are important prerequisites.

Since the submission of this paper, Chandra et al. ${ }^{3}$ have described their experience with endoscopic hemispherotomy. Unlike the technique that we present in this paper, they have used a 3-handed approach (Fig. 5) similar to one
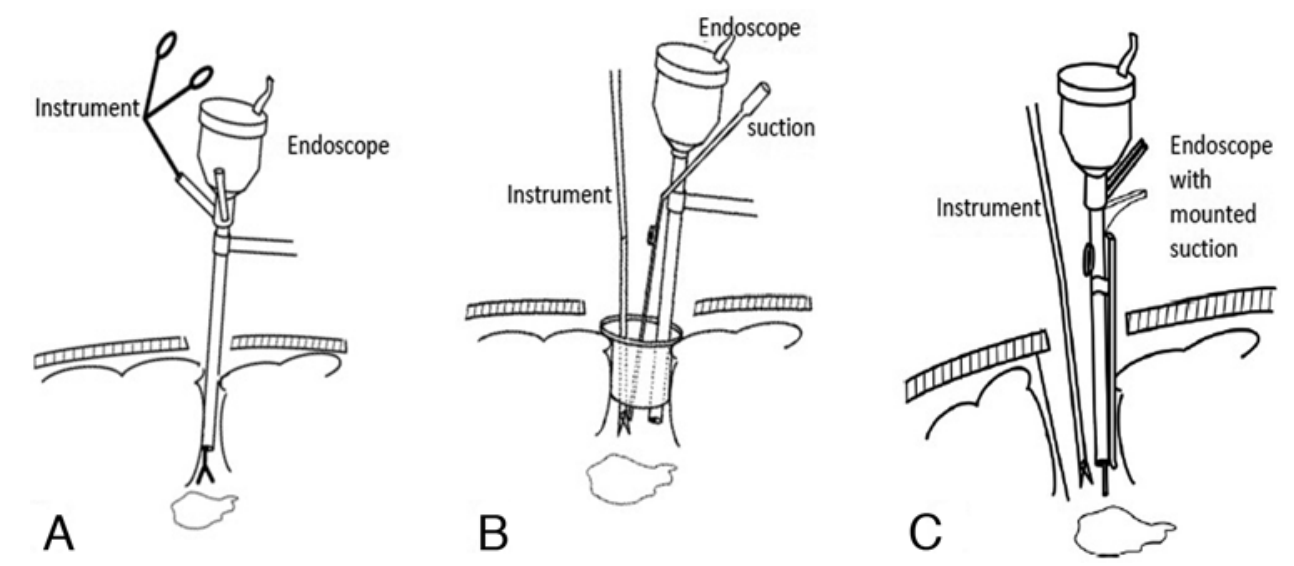

FIG. 5. Drawings showing the various techniques. A: One-handed technique performed using an endoscope through a bur hole. B: Three-handed technique performed using an endoscope through a microcraniotomy. An assistant or a mechanical device holds the endoscope, and 2 hands are used for conducting surgery. C: Bimanual technique in which the endoscope with mounted suction is used in one hand and another instrument is in the other hand, for bimanual operation. Copyright Sandeep Sood. Published with permission. 
used in traditional endoscopic transsphenoidal surgery. For parts requiring bimanual dissection, the endoscope is either "supported by an assistant or held with a holding device." As discussed above, this not only requires a larger exposure but also the nuisance of repeatedly readjusting the position of the endoscope if held on a holder, or the crowding of hands if held by an assistant. In addition, these practitioners have used the sphenoid ridge and planum sphenoidale as a landmark for anterior resection. Using the ACA as we have offers a distinct advantage in that it ensures that there is no residual posterior frontal basal cortex left in connection with the thalamus.

\section{Conclusions}

Endoscopic disconnective procedures are an attractive option to an open operation. However, the use of standard 2D endoscopes does involve a learning curve. We are excited about adapting the recently introduced 3D endoscope to these operations, retaining the advantages of depth visualization of microscopic surgery, small exposures, and the greater magnification obtained with an endoscope.

\section{References}

1. Bahuleyan B, Manjila S, Robinson S, Cohen AR: Minimally invasive endoscopic transventricular hemispherotomy for medically intractable epilepsy: a new approach and cadaveric demonstration. J Neurosurg Pediatr 6:536-540, 2010

2. Bahuleyan B, Vogel TW, Robinson S, Cohen AR: Endoscopic total corpus callosotomy: cadaveric demonstration of a new approach. Pediatr Neurosurg 47:455-460, 2011

3. Chandra PS, Kurwale N, Garg A, Dwivedi R, Malviya SV, Tripathi M: Endoscopy-assisted interhemispheric transcallosal hemispherotomy: preliminary description of a novel technique. Neurosurgery 76:485-495, 2015

4. Cutler AR, Kaloostian SW, Ishiyama A, Frazee JG: Twohanded endoscopic-directed vestibular nerve sectioning: case series and review of the literature. J Neurosurg 117:507-513, 2012

5. Delalande O, Bulteau C, Dellatolas G, Fohlen M, Jalin C, Buret V, et al: Vertical parasagittal hemispherotomy: surgical procedures and clinical long-term outcomes in a population of 83 children. Neurosurgery 60:ONS19ONS32, 2007

6. Guerrero MH, Cohen AR: Endoscope-assisted microsurgery of the corpus callosum. Minim Invasive Neurosurg 46:5456,2003

7. Harris AE, Hadjipanayis CG, Lunsford LD, Lunsford AK, Kassam AB: Microsurgical removal of intraventricular lesions using endoscopic visualization and stereotactic guidance. Neurosurgery 62 (Suppl 2):622-629, 2008
8. Iwasaki M, Uematsu M, Sato Y, Nakayama T, Haginoya K, Osawa S, et al: Complete remission of seizures after corpus callosotomy. J Neurosurg Pediatr 10:7-13, 2012

9. Kasasbeh AS, Smyth MD, Steger-May K, Jalilian L, Bertrand M, Limbrick DD: Outcomes after anterior or complete corpus callosotomy in children. Neurosurgery 74:17-28, 2014

10. Kassam AB, Snyderman C, Gardner P, Carrau R, Spiro R: The expanded endonasal approach: a fully endoscopic transnasal approach and resection of the odontoid process: technical case report. Neurosurgery 57:E213, 2005

11. Sood S, Hoeprich M, Ham SD: Pure endoscopic removal of pineal region tumors. Childs Nerv Syst 27:1489-1492, 2011

12. Sood S, Nundkumar N, Ham SD: Interhemispheric endoscopic resection of large intraventricular and thalamic tumors. J Neurosurg Pediatr 7:596-599, 2011

13. Tubbs RS, Smyth MD, Salter G, Doughty K, Blount JP: Eyebrow incision with supraorbital trephination for endoscopic corpus callosotomy: a feasibility study. Childs Nerv Syst 20:188-191, 2004

\section{Disclosure}

The authors report no conflict of interest concerning the materials or methods used in this study or the findings specified in this paper.

\section{Author Contributions}

Conception and design: Sood. Acquisition of data: Sood, Asano. Analysis and interpretation of data: Sood, Marupudi. Drafting the article: Sood, Marupudi. Critically revising the article: all authors.

\section{Supplemental Information \\ Videos}

Video 1, Media Player. http://mfile.akamai.com/21490/wmv/ digitalwbc.download.akamai.com/21492/wm.digitalsource-naregional/peds15-31_video_1.asx.

Video 1, Quicktime. http://mfile.akamai.com/21488/mov/ digitalwbc.download.akamai.com/21492/qt.digitalsource-global/ peds15-31_video_1.mov.

\section{Previous Presentation}

This paper was presented at the 43rd Annual Meeting of the AANS/CNS Section on Pediatric Neurological Surgery, which was held December 2-5, 2014, on Amelia Island, Florida.

\section{Correspondence}

Sandeep Sood, Pediatric Neurosurgery, Children's Hospital of Michigan, 3901 Beaubien, 2nd Fl., Detroit, MI 48201. email: ssood@med.wayne.edu. 